\title{
Loss of Coherence of Low Frequency Fluctuations of BOLD FMRI in Visual Cortex of Healthy Aged Subjects
}

\author{
Lirong Yan ${ }^{1,2}$, Yan Zhuo ${ }^{1}$, Bo Wang ${ }^{1}$ and Danny J.J. Wang*,2 \\ ${ }^{I}$ Beijing MRI Center for Brain Research, Institute of Biophysics, Chinese Academy of Sciences, Beijing, China; \\ ${ }^{2}$ Ahmanson-Lovelace Brain Mapping Center, Department of Neurology, UCLA, Los Angeles, CA, USA
}

\begin{abstract}
Aging effects on blood-oxygen-level-dependent (BOLD) functional MRI (fMRI) have been studied using task induced hemodynamic responses with controversial findings. The present study systematically investigated the normal aging effect in the visual cortex using 3 indices of low frequency fluctuations of resting state BOLD fMRI, i.e., amplitude of low frequency fluctuations (ALFF), regional homogeneity (ReHo) and functional connectivity (FC). These 3 resting state measurements were compared with task induced BOLD activation in the visual cortex of 2 groups of 10 young and 10 elderly subjects. Our results showed reduced functional connectivity and regional homogeneity of low frequency fluctuations of BOLD fMRI in aged subjects as compared to young subjects. While the mean magnitude of BOLD activation and the mean amplitude of low frequency fluctuations of BOLD fMRI did not vary between the 2 age groups, larger variances were observed in both measures in aged subjects. These data suggest that normal aging may be associated with "loss of coherence" of low frequency fluctuations of resting state BOLD fMRI in the visual cortex, and may affect task induced BOLD response through increased inter- and intra-subject variability.
\end{abstract}

Keywords: Blood-Oxygen-Level-Dependent (BOLD); Aging; Amplitude of Low Frequency Fluctuations (ALFF); Regional Homogeneity (ReHo); Functional Connectivity (FC).

\section{INTRODUCTION}

Functional magnetic resonance imaging (fMRI) based on blood oxygen level dependent (BOLD) contrast has been increasingly applied for studying neurodegenerative disorders such as dementia, Parkinson's disease and multiple sclerosis etc [1-3]. However, the "normal" aging effect on human cerebrovascular system and brain function needs to be understood thoroughly before clinical BOLD fMRI studies on neurodegenerative diseases can yield meaningful results regarding the underlying pathophysiology.

Several studies have investigated the relationship of BOLD fMRI and normal aging [4-11], typically through comparisons of the magnitude of the hemodynamic response function (HRF) elicited by a standard motor or visual task between young and elderly subjects. Somewhat controversial results have been reported. Some studies observed that the amplitude of the BOLD response is reduced in elderly subjects $[4,6,11,12]$, whereas others failed to detect significant differences [7, 8]. Observed differences in the magnitude of the BOLD response may be sensitive to the selection of region-of-interest (ROI) or activated voxels [8]. Several studies have also observed that elderly subjects have higher noise levels in activated voxels, resulting in lower signal-to-noise ratio (SNR) [6, 7]. Moreover, some studies have observed that elderly subjects show increased inter- and intra-subject variability in the hemodynamic response as compared to young subjects [7].

*Address correspondence to this author at the UCLA Department of Neurology, Ahmanson-Lovelace Brain Mapping Center, 660 Charles E. Young Drive South, Los Angeles, CA 90095-7085, USA;

Tel; 310-983-3667; 310-794-7406; E-mail: jwang71@gmail.com
Normal aging is typically associated with cognitive decline, accompanied by progressive slowness and impaired motor ability $[13,14]$. For BOLD fMRI studies on aging using sensorimotor and cognitive tasks, the observed differences in BOLD responses may be confounded by deteriorating performance as well as compensatory processes in aged subjects. Recent studies have shown that low frequency temporal components $(<0.1 \mathrm{~Hz})$ in resting state BOLD fMRI reflect spontaneous fluctuations of brain physiology and metabolism [15], which may serve as imaging markers of brain function without the use of external tasks. Several indices of spontaneous low frequency fluctuations of BOLD fMRI have been introduced to characterize resting brain function, such as the amplitude of low frequency fluctuations (ALFF) [16, 17], regional homogeneity (ReHo) and functional connectivity (FC) $[18$, 19]. The latter two indices calculate the "coherence" or correlation of low frequency fluctuations of BOLD fMRI between adjacent voxels and across all brain voxels, respectively. Although the exact underlying biophysical mechanisms remain unclear, a few studies have applied FC and $\mathrm{ReHo}$ of low frequency fluctuations of BOLD fMRI to characterize healthy aging effects [18-20]. These studies have consistently reported reduced FC and ReHo with aging, suggesting that resting state BOLD fMRI may provide more reliable and sensitive markers of aging effects than task induced BOLD responses.

To date, however, there are few BOLD fMRI studies of the aging effect that systematically investigated the above three resting state measurements (ALFF, ReHo and FC) as 
well as their associations with task-induced BOLD response. In the present study, we attempted to fill in this knowledge gap by applying all three indices of low frequency fluctuations of resting state BOLD fMRI which were further compared with visual cortex activation in two groups of healthy young and elderly volunteers. Our hypothesis was that normal aging is associated with reduced coherence of resting state BOLD fMRI as manifested by decreased functional connectivity and regional homogeneity. We also hypothesize that normal aging is associated with reduced BOLD responses to sensorimotor activation as well as reduced amplitude of low frequency fluctuations of BOLD fMRI at baseline.

\section{METHODS}

\section{Experimental Design and Imaging Parameters}

All MR imaging experiments were performed on a Siemens Tim Trio 3T scanner (Erlangen, Germany) using 12-channel head coil. A total of 20 subjects (Chinese Han ethnicity) including 10 young volunteers (age $23 \pm 2$ yrs, 6 males) and 10 elderly subjects (age $66 \pm 3$ yrs, 5 males) participated in this study after they provided written informed consent. All participants were screened for neurological or psychiatric illnesses including alcoholism, depression, schizophrenia, bipolar affective disorder, prior history of stroke or significant head injury, Alzheimer's, Parkinson's, or Huntington's disease [8]. A single-shot dualecho gradient-echo EPI with interleaved TE was developed to acquire 4 different TE data sets for every 2 consecutive TRs $(\mathrm{TE} 1=20 \mathrm{~ms}$ and TE $2=50 \mathrm{~ms}$ for one TR, TE $3=35 \mathrm{~ms}$ and TE4 $=65 \mathrm{~ms}$ for the following TR) [17]. The purpose of this multiecho EPI sequence was to account for potential changes of T2* between the 2 age groups. Each subject underwent two experiments within the same scanning session using the dual-echo EPI sequence: 1) resting state with eyes closed; and 2) block-design 30s off/on flashing checkerboard visual stimulation. Each scan with 480 acquisitions took $8 \mathrm{~min}$. Ten oblique slices with $5 \mathrm{~mm}$ thickness and $1 \mathrm{~mm}$ gap were scanned parallel to the anterior-posterior commissure (ACPC). Other parameters included: $\mathrm{FOV}=220 \mathrm{~mm}$; matrix $=64 \times 64$; bandwidth $=2442 \mathrm{~Hz} /$ pixel; $\mathrm{TR}=1 \mathrm{~s}$ (effective $\mathrm{TR}=2 \mathrm{~s}$ ); flip angle $=65^{\circ}$. To remove the contribution of cardiac and respiratory pulsation in the spontaneous low frequency fluctuations of BOLD fMRI, respiratory and cardiac pulses were recorded in real time during all experiments using the scanner's built-in pulse-oximetry and a respiratory belt, respectively. For each subject, conventional $\mathrm{T} 1$ weighted $3 \mathrm{D}$ images were acquired using an MPRAGE sequence (TR/TE/TI=1730/ 3.96/ $1100 \mathrm{~ms}$; flip angle $=15^{\circ}$; matrix $=128 \times 128$, voxel size $=1.9 \times 1.9 \times 1.9 \mathrm{~mm}^{3}$ ) for anatomic MRI.

\section{Data Processing}

For each subject, all EPI data were corrected for head motion offline using SPM2 (Wellcome Department ofImaging Neuroscience, University College London), which was performed independently for each TE. Image registration was also performed to align the resting and activation scans. Analyses of fMRI data were carried out at individual subject level and group level.
For individual subject analyses, no spatial smoothing was applied since previous studies have shown that spatial smoothing may modulate (enhance) the magnitude of low frequency noise in BOLD fMRI $[21,22]$. During the resting state, for each brain pixel, the amplitude of low frequency fluctuations (ALFF) was determined by the standard deviation (SD) of the signal time course in the low frequency band of $0 \sim 0.1 \mathrm{~Hz}$ (normalized by mean intensity). BOLD fMRI signals associated with recorded respiratory and cardiac pulsations were removed offline using retrospective image-based correction (RETROICOR) [23]. Brain activation data were analyzed using general linear model in SPM2. The $Z$ statistic maps were thresholded at a significance level of $p<10^{-2}$ with at least 20 contiguous voxels for each TE dataset in each subject. For each subject, the ROI (here named as sROI) was defined by the pixels demonstrating common activation for all TE data in the visual cortex. The task-induced BOLD signal changes and low frequency fluctuations were drawn from sROI for the comparison between the two age groups. Furthermore, the relationship between task-induced BOLD signal changes and low frequency fluctuations was investigated based on mean values within sROI across subjects.

For functional connectivity analyses of the resting state fMRI, the seed was defined to include all significant voxels within $6 \mathrm{~mm}$ of the location of the peak activation in each subject. Correlation analysis was then carried out across brain pixels using the seed time course as the reference function. To study the regional homogeneity (ReHo) of resting state fMRI between the two age groups, we adopted Kendall's coefficient of concordance (KCC) $[18,24]$ to measure regional homogeneity of the time series of a givenvoxel with those of its nearestneighbors (26 voxels) in a voxel-wise manner.

For group analyses of BOLD activation, image normalization into a canonical template (Montreal Neurology Institute brain atlas) and spatial smoothing (FWHM $=6 / 6 / 8 \mathrm{~mm}$ ) were performed in each subject, followed by one-sample t-test within the young and aged group respectively. Two subjects (a young subject and an elderly subject) were excluded from group analyses, because of obvious failure of spatial normalization. The statistic map for each subject was also thresholded at a significance level of $\mathrm{p}<10^{-2}$ with at least 20 contiguous voxels for each TE dataset. The number of activated pixels and mean $T$ values for each subject were compared between the two age groups. The group FC and ReHo maps were generated by performing one-sample t-test on the spatially normalized FC and ReHo maps of all subjects in each age group, respectively. Mean values of BOLD signal changes, ALFF, $\mathrm{FC}$ and $\mathrm{KCC}$ were measured from 2 visual ROIs respectively: 1) common ROI (cROI) including activated voxels in both the young and elderly groups during the activation fMRI scan; and 2) or ROI (oROI) including activated voxels in either the young or elderly group during the activation fMRI scan.

\section{Statistical Analysis}

Statistical analyses were performed using the SPSS 13.0 software package (SPSS, Inc., Chicago, IL, USA). The repeated measures analysis of variance (ANOVA) was 


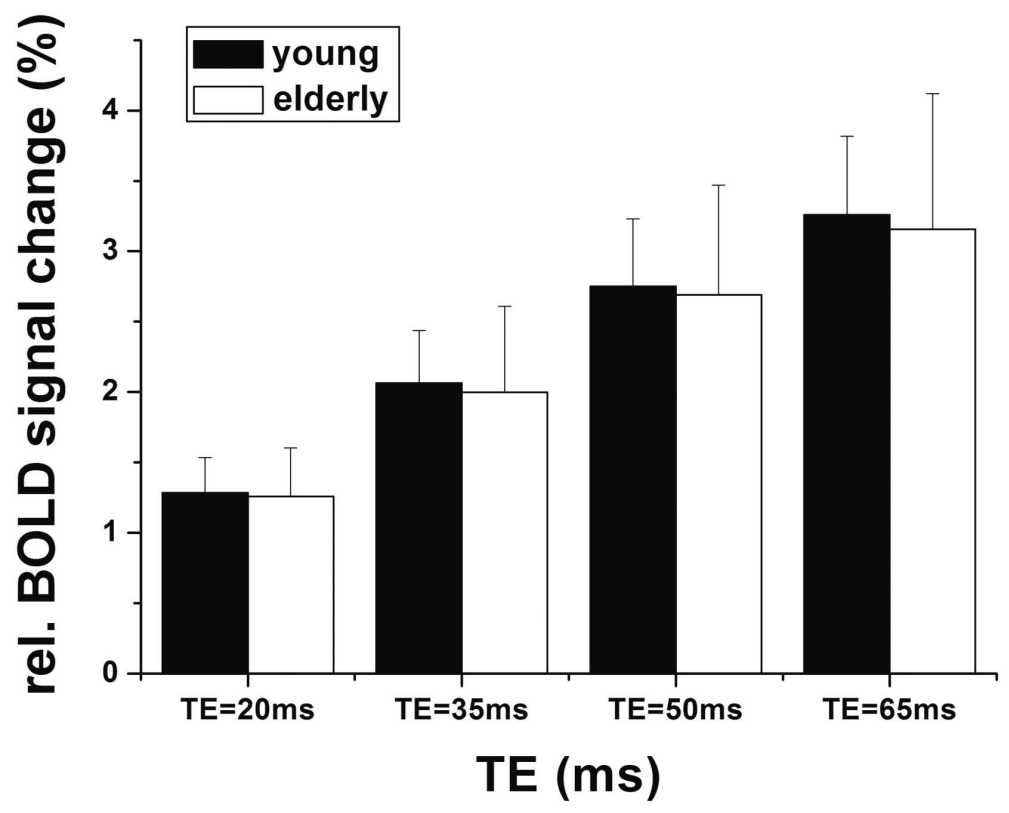

Fig. (1). Mean relative BOLD signal changes between the young and aged subjects within sROI (the individual visual activated ROIs) at each TE.

applied to test the effect of TE and aging on the BOLD signal changes, ALFF, ReHo and FC. Pearson correlation coefficients $(r)$ were used to evaluate associations between ALFF, ReHo, FC and BOLD signal changes, respectively. The Wilcoxon rank sum test was used for comparison of $T$ and $r$ values between the two age groups. The standard deviations (SD) of activation and resting fMRI measurements were compared using F-test.

\section{RESULTS}

\section{Comparison of BOLD Activation Between Two Groups}

Fig. (1) shows the mean signal changes drawn from visual sROI of each subject between the two age groups at 4 different TEs. As expected, the BOLD signal changes show significant dependence on TE $(\mathrm{p}<0.001)$. The mean $\mathrm{T} 2 *$ values were not different between the young $(\mathrm{T} 2 *=$ $51.21 \pm 2.63 \mathrm{~ms})$ and elderly $(\mathrm{T} 2 *=51.19 \pm 2.70 \mathrm{~ms})$ subjects. There was no significant difference of BOLD signal changes between the two age groups $(p=0.60)$. Fig. (1) also shows that the SD of BOLD signal changes of the elderly subjects was larger than that of the young group, and the statistical analysis showed an trend toward significance $(p=0.078)$. As a result of the increased inter-subject variability in elderly subjects, the young group showed more activated pixels than those of the elderly subjects $(p=0.01)$ in the normalized activation maps. However, there was no significant difference in the mean T-value $(\mathrm{p}=0.96)$ and peak T-value $(p=0.35)$ between the two age groups. Fig. (2) shows the group activation maps in the young and aged groups at the representative TE of $35 \mathrm{~ms}$. It can be clearly seen that the area of activation in the young group (pixel no. $=4626$, peak $\mathrm{T}$ value $=16.22$, mean $\mathrm{T}$ value $=6.73$ ) was larger than that in the aged group (pixel no. $=3017$,peak $\mathrm{T}$ value $=18.93$, mean $\mathrm{T}$ value $=6.39$ at $T E=35 \mathrm{~ms}$ ). In addition, the mean values of BOLD signal changes were not significantly different in either the cROI or oROI ( $p>0.13)$ between the young and elderly groups.

\section{Comparison of Measurements of Low Frequency Fluctuations Between Two Groups}

Fig. (3) shows the magnitude of low frequency fluctuations (ALFF) of the young and elderly groups within the sROI at 4 different TEs. There is significant dependence of ALFF on TE $(p<0.001)$, but no significant difference between the two age groups at each TE $(p=0.95)$. The SD of ALFF of the elderly subjects was significantly larger than that of the young group, suggesting increased inter-subject variability in elderly subjects $(\mathrm{p}=0.014)$. In group analyses, the mean values of ALFF were not significantly different in either cROI or oROI ( $p>0.38)$ between the young and elderly groups. This result of ALFF is similar to that of activation induced BOLD signal changes. Fig. (4) shows the group map of functional connectivity analysis in the 2 age groups. Functional connectivity within the visual oROI was stronger in the young group than that of the aged group $(\mathrm{p}=0.056,1$ tailed, mean $\mathrm{r}=0.70 \pm 0.13$ for young subjects and $\mathrm{r}=0.58 \pm 0.15$ for elderly subjects). Fig. (5) shows the mean KCCs in the visual oROIs between the two groups at 4 different TEs, which is an index of intra-subject regional homogeneity of resting state BOLD fMRI. There were significant differences $(\mathrm{p}=0.029,1$-tailed $)$ for mean regional homogeneity values between the 2 age groups in the visual oROI. However, mean FC and ReHo values were not significantly different $(\mathrm{p}>0.14)$ in the cROI including commonly activated pixels in the young and elderly groups during visual cortex activation.

\section{The Relationship Between BOLD Signal Changes and Low Frequency Fluctuations}

Fig. (6a) and (b) show the scatter plots of the mean BOLD signal changes and the mean magnitude of the low frequency fluctuations in the visual sROIs of the young and elderly group, respectively. We found that there were significant linear correlations between the BOLD response and the magnitude of low frequency fluctuations in both age 


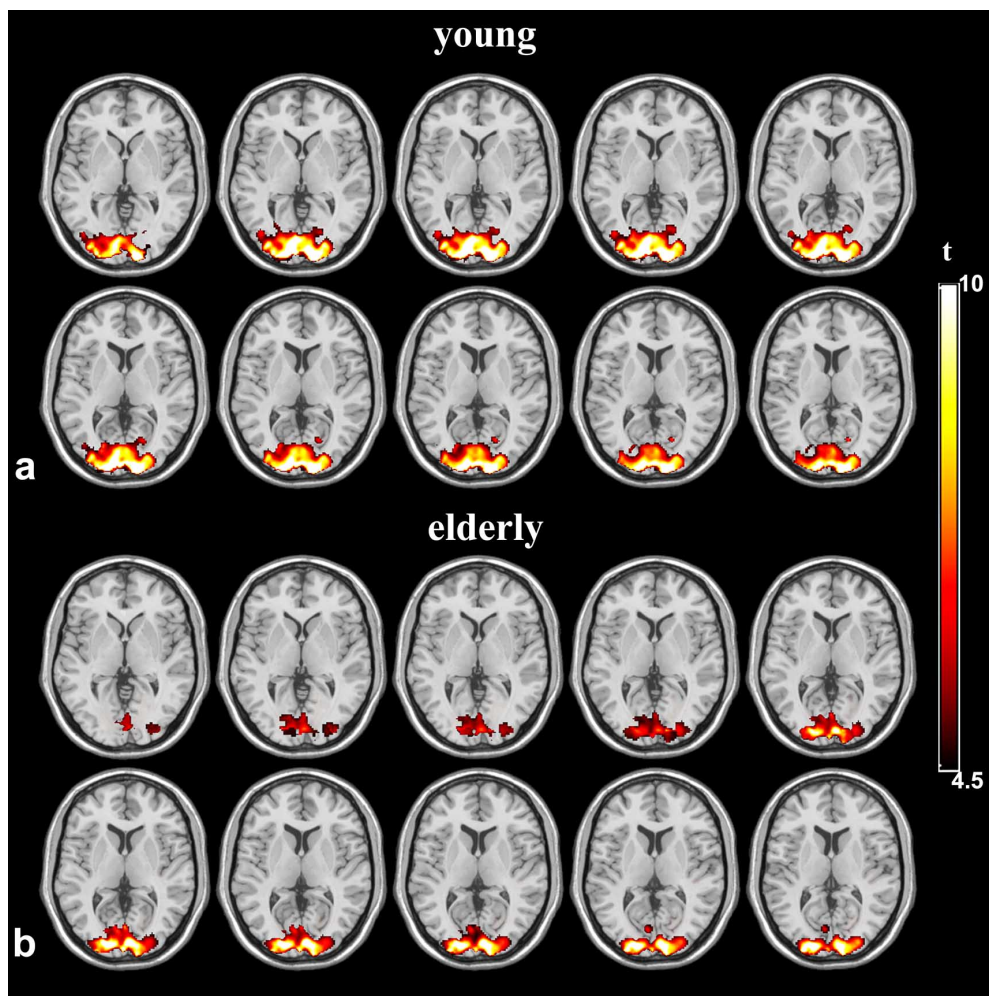

Fig. (2). Group activation statistical maps between young and elderly groups at $\mathrm{TE}=35 \mathrm{~ms}$

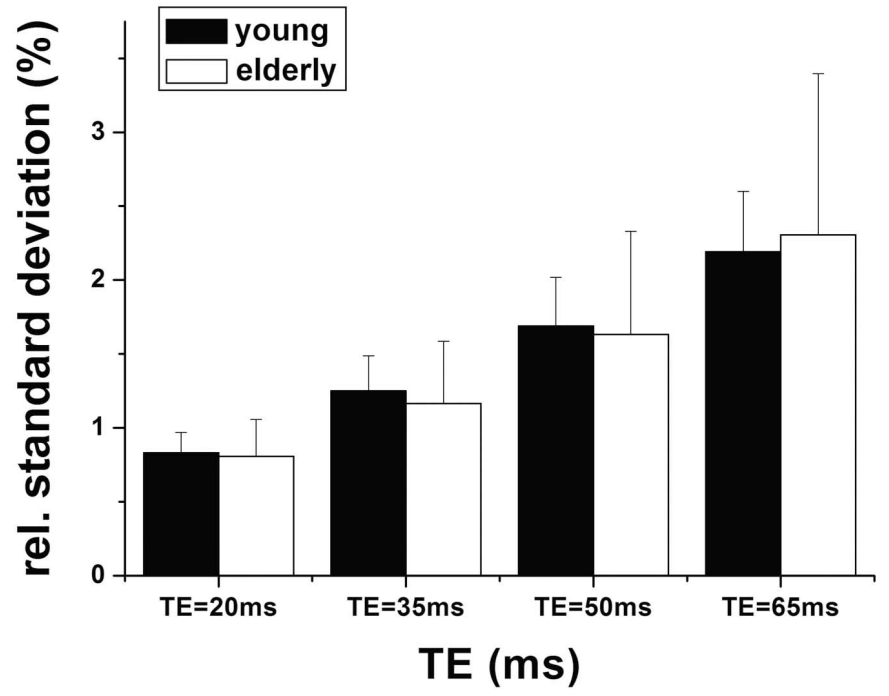

Fig. (3). Mean amplitudes of low frequency fluctuations between the young and aged subjects within sROI (the individual visual activated ROIs) at each TE.

groups (young subjects: $\mathrm{r}=0.82, \mathrm{p}<0.01$; elderly subjects: $\mathrm{r}=0.56, \mathrm{p}<0.01)$. The scatter plot in elderly subjects showed larger variances/dispersion compared to young subjects, however no significant difference $(p=0.33)$ was detected between the two groups. No significant associations between ReHo and BOLD activation, or between FC and BOLD activation were detected across subjects.

\section{DISCUSSION}

In this study, we investigated healthy aging effect on BOLD responses to visual stimulation, as well as the aging effect on 3 measurements of low frequency fluctuations during the resting state in the visual cortex. We further investigated the associations between BOLD activation and low frequency fluctuations at baseline in the two age groups. We found that mean BOLD signal changes in response to visual stimulation was not significantly different between the two age groups, which was consistent with several earlier BOLD fMRI studies on aging [7, 8]. In previous fMRI studies on aging, controversial results about the BOLD response have been observed. One potential explanation is that the observed group differences in the magnitude of BOLD responses may be sensitive to voxel selection or ROI definition [8]. For instance, although mean BOLD signal 


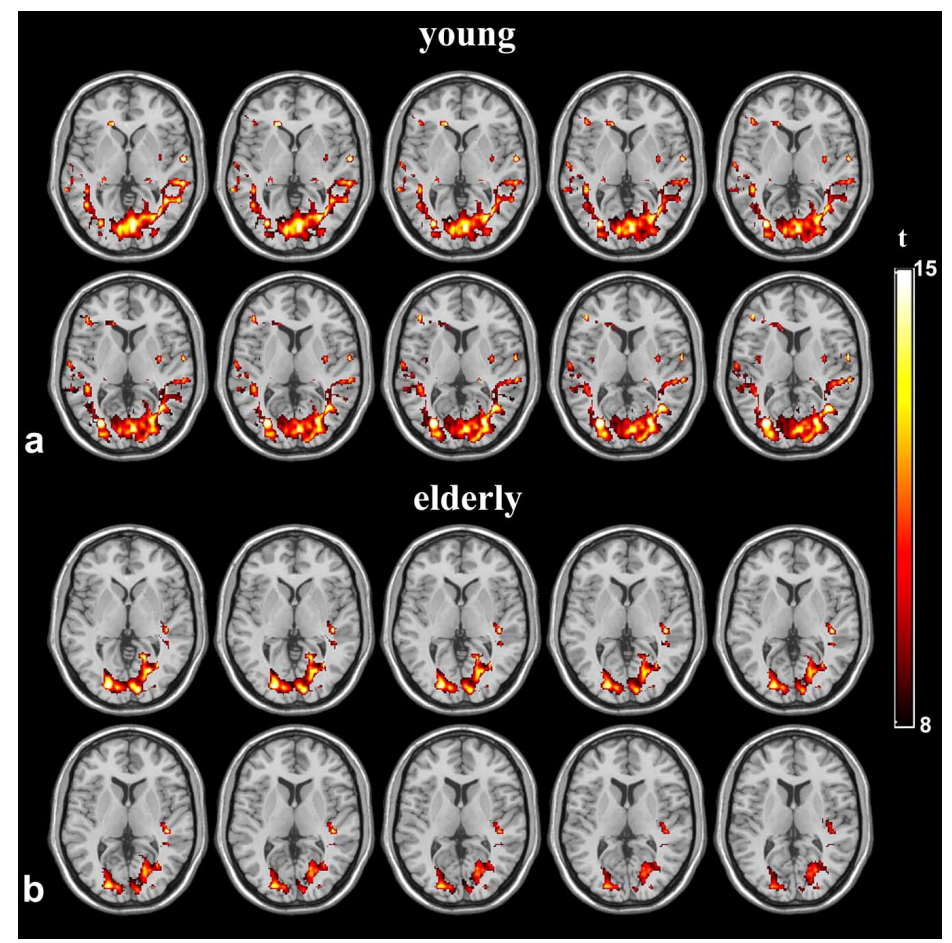

Fig. (4). Group functional connectivity maps of young and elderly groups at $\mathrm{TE}=35$.

changes are not different in pixels of peak activation between young and elderly subjects, the number of "deactivated" pixels and thereby the variance of BOLD activation increase in aged subjects when larger ROIs are used. Overall, past fMRI studies on aging seem to suggest both increased interand intra-subject variability of task induced BOLD responses in aged subjects, although the mean magnitude of peak activation may not be significantly affected by age. Our findings are largely consistent with previous fMRI studies since the area of group activation was larger in young subjects compared to elderly subjects, while mean BOLD signal changes were not different between the 2 age groups. Additionally, the inter-subject variability (SD) of BOLD activation was larger in the elderly subjects than young subjects, as demonstrated in Fig. 1.

A consensus has emerged from recent studies that low frequency fluctuations of resting state BOLD fMRI reflect spontaneous fluctuations in brain physiology and metabolism at baseline. Measurements of resting state BOLD fMRI may provide surrogate indices of baseline brain function without the use of external tasks. In the present study, we systematically investigated and compared 3 indices of low frequency fluctuations of BOLD fMRI (ALFF, ReHo and FC) in characterizing aging effects in the visual cortex. The main findings were: 1) there was no difference in mean ALFF values between the two age groups, but the intersubject variability of ALFF was larger in elderly subjects than that of young subjects $(\mathrm{p}=0.014)$. This result matches well with activation induced BOLD fMRI data; 2) The group FC map (Fig. 4) demonstrates weaker FC in the visual cortex (oROI) of elderly subjects than that of young subjects $(\mathrm{p}=0.056)$; 3) The mean ReHo values were reduced in the visual cortex (oROI) of aged subjects compared to young subjects $(\mathrm{p}=0.029)$. All 3 findings provided converging evidence supporting the hypothesis for the "loss of coherence" in low frequency fluctuations of BOLD fMRI with aging. In particular, both $\mathrm{ReHo}$ and $\mathrm{FC}$ are direct indices of regional and long-range coherence of spontaneous fluctuations of BOLD fMRI. Reductions of FC and ReHo have been reported in aged subjects and patients with Parkinson's and Alzheimer's disease [18, 19, 25, 26], suggesting measurements of resting state BOLD fMRI may provide sensitive and reliable imaging markers of healthy aging and neurodegenerative processes while avoiding confounding factors such as deteriorating performance and compensatory processes etc.

It is worth noting that the differences in FC and ReHo values were detected in the relative large oROI including activated pixels in either the young or the aged group rather than the cROI that contained commonly activated pixels. This result suggests that the observed loss of coherence of low frequency fluctuations (reduced $\mathrm{ReHo}$ and FC) with healthy aging is a quite subtle effect, at least in the visual cortex. It is manifested in the peripheries of the activation foci where larger variances of BOLD responses have been reported in aged volunteers [8]. The biophysical mechanisms underlying age-related loss of coherence of low frequency fluctuations of resting state BOLD fMRI remain unclear. Because both parameters measure the similarity of the time series of a given voxel to those of its nearest neighbors and to those of all brain pixels, ReHo and FC are likely mediated by short and long range cortical connections. Loss or malfunction of these connections may play a role. It has been reported that aging may affect cortical and sub-cortical connections through cell loss, synaptic degeneration, blood flow reduction,neurochemical alteration as well as central nervous system reorganization [27]. All these histological changes may influence regional homogeneity and functional connectivity in the visual cortex as well as in other brain regions. 


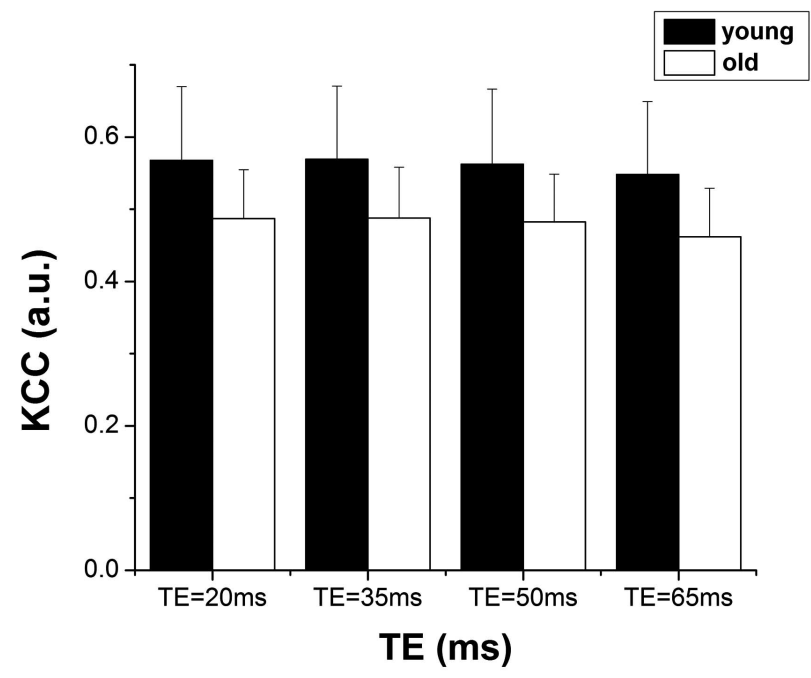

Fig. (5). Mean KCC between the young and elderly subjects within the oROI containing activated pixels in either the young or aged group at each TE.
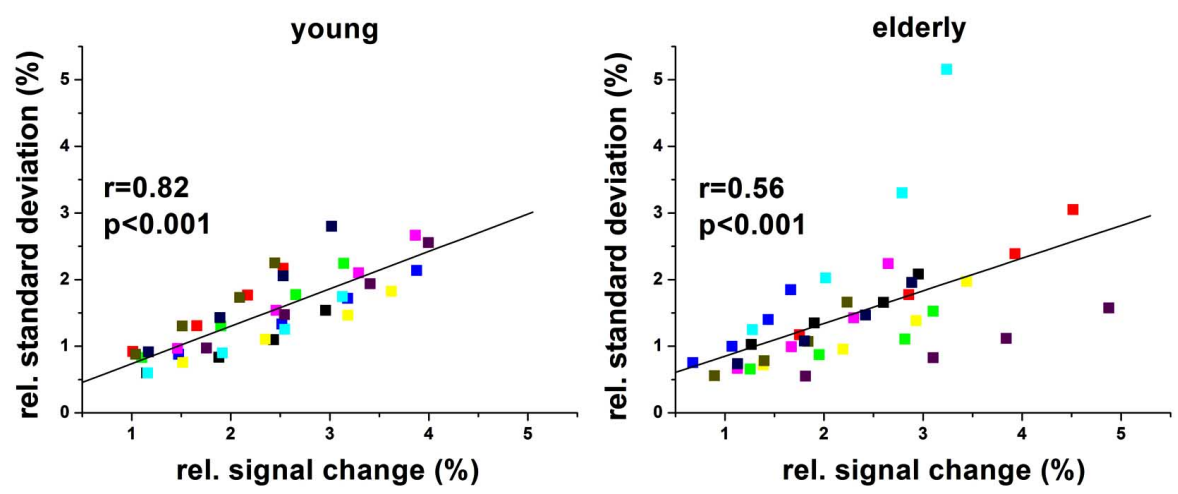

Fig. (6). Scatter plot of amplitude of low frequency fluctuations (ALFF $<0.1 \mathrm{~Hz}$ ) vs.relative BOLD signal changes in the visual sROIs for young and elderly groups. Each color represents one subject.

Another finding from the present study as well as earlier studies $[17,28]$ was significant linear correlations between activation induced BOLD signal changes and the magnitude of low frequency fluctuations at baseline $(<0.1 \mathrm{~Hz})$. This observation suggests a shared mechanism underlying the BOLD contrast and spontaneous fluctuations in BOLD fMRI, such as aerobic glycolysis [29]. The apparently increased variances/dispersion of associations between activation induced BOLD signal changes and ALFF in aged subjects is not well understood (Fig. 6). It may be related to greater variances of vascular reactivity in aged compared to young subjects, which can be assessed using fMRI responses to hypercapnia challenge. The lack of associations between ReHo, FC and activation induced BOLD responses suggests that $\mathrm{ReHo}$ and $\mathrm{FC}$ may reflect different aspects and properties of the resting state fMRI compared to ALFF. It is highly likely that ReHo, FC and ALFF together may provide thorough characterization of the resting state brain function than using each parameter alone. In the present study, the image coverage was limited (10 6mm slices parallel to ACPC covering the visual cortex) due to acquisition of multi-TE fMRI data. Our findings need to be replicated and validated in brain regions other than the visual cortex (e.g. default network) in future studies on aging and neurodegenerative diseases.

\section{CONCLUSION}

The present study systematically investigated the normal aging effect using 3 indices of low frequency fluctuations of resting state BOLD fMRI, and compared them with task induced BOLD activation in the visual cortex. Our results showed reduced functional connectivity and regional homogeneity of low frequency fluctuations of BOLD fMRI in aged subjects as compared to young subjects. While the mean magnitude of BOLD activation and the mean amplitude of low frequency fluctuations of BOLD fMRI did not vary between the 2 age groups, larger variabilities were observed in both measures in aged subjects. These data suggest that normal aging may be associated with loss of coherence of low frequency fluctuations of resting state BOLD fMRI in the visual cortex, and may affect task induced BOLD response through increased inter- and intrasubject variability.

\section{ACKNOWLEDGEMENT}

This work was supported by Ministry of Science and Technology of China grant (2005CB522800), National Nature Science Foundation of China grants (30621004,90820307) and US NIH grant AG016570-11A. 


\section{REFERENCES}

[1] Rombouts SA, van Swieten JC, Pijnenburg YA, Goekoop R, Barkhof F, Scheltens P. Loss of frontal fMRI activation in early frontotemporal dementia compared to early AD. Neurology 2003; 60(12): 1904-8.

[2] Tada Y. Motor association cortex activity in Parkinson's disease--a functional MRI study. Rinsho Shinkeigaku 1998; 38(8): 729-35.

[3] Buckle GJ. Functional magnetic resonance imaging and multiple sclerosis: the evidence for neuronal plasticity. J Neuroimag 2005; 15(4 Suppl): 82S-93S.

[4] Ross MH, Yurgelun-Todd DA, Renshaw PF, et al. Age-related reduction in functional MRI response to photic stimulation. Neurology 1997; 48(1): 173-6.

[5] D'Esposito M, Zarahn E, Aguirre GK, Rypma B. The effect of normal aging on the coupling of neural activity to the bold hemodynamic response. Neuroimage 1999; 10(1): 6-14.

[6] Buckner RL, Snyder AZ, Sanders AL, Raichle ME, Morris JC. Functional brain imaging of young, nondemented, and demented older adults. J Cogn Neurosci 2000; 12 (Suppl 2): 24-34.

[7] Huettel SA, Singerman JD, McCarthy G. The effects of aging upon the hemodynamic response measured by functional MRI. Neuroimage 2001; 13(1): 161-75.

[8] Aizenstein HJ, Clark KA, Butters MA, et al. The BOLD hemodynamic response in healthy aging. J Cogn Neurosci 2004; 16(5): 786-93.

[9] Hesselmann V, Zaro Weber O, Wedekind C, et al. Age related signal decrease in functional magnetic resonance imaging during motor stimulation in humans. Neurosci Lett 2001; 308(3): 141-4.

[10] Mattay VS, Fera F, Tessitore A, et al. Neurophysiological correlates of age-related changes in human motor function. Neurology 2002; 58(4): 630-5.

[11] Raemaekers M, Vink M, van den Heuvel MP, Kahn RS, Ramsey NF. Effects of aging on BOLD fMRI during prosaccades and antisaccades. J Cogn Neurosci 2006; 18(4): 594-603.

[12] Tekes A, Mohamed MA, Browner NM, Calhoun VD, Yousem DM. Effect of age on visuomotor functional MR imaging. Acad Radiol 2005; 12(6): 739-45.

[13] Woodard JL, Seidenberg M, Nielson KA, et al. Prediction of Cognitive Decline in Healthy Older Adults using fMRI. J Alzheimers Dis 2010; 21(3): 871-85.

[14] Smith CD, Umberger GH, Manning EL, et al. Critical decline in fine motor hand movements in human aging. Neurology 1999; 53(7): 1458-61.
[15] Fox MD, Raichle ME. Spontaneous fluctuations in brain activity observed with functional magnetic resonance imaging. Nat Rev Neurosci 2007; 8(9): 700-11.

[16] Yang H, Long XY, Yang Y, et al. Amplitude of low frequency fluctuation within visual areas revealed by resting-state functional MRI. Neuroimage 2007; 36(1): 144-52.

[17] Yan L, Zhuo Y, Ye Y, et al. Physiological origin of low-frequency drift in blood oxygen level dependent (BOLD) functional magnetic resonance imaging (fMRI). Magn Reson Med 2009; 61(4): 819-27.

[18] Wu T, Zang Y, Wang L, Long X, Li K, Chan P. Normal aging decreases regional homogeneity of the motor areas in the resting state. Neurosci Lett 2007; 423(3): 189-93.

[19] Wu T, Zang Y, Wang L, et al. Aging influence on functional connectivity of the motor network in the resting state. Neurosci Lett 2007; 422(3): 164-8.

[20] Damoiseaux JS, Beckmann CF, Arigita EJ, et al. Reduced restingstate brain activity in the "default network" in normal aging. Cereb Cortex 2008; 18(8): 1856-64.

[21] Aguirre GK, Zarahn E, D'Esposito M. Empirical analyses of BOLD fMRI statistics. II. Spatially smoothed data collected under nullhypothesis and experimental conditions. Neuroimage 1997; 5(3): 199-212.

[22] Wang J, Wang Z, Aguirre GK, Detre JA. To smooth or not to smooth? ROC analysis of perfusion fMRI data. Magn Reson Imaging 2005; 23(1): 75-81.

[23] Glover GH, Li TQ, Ress D. Image-based method for retrospective correction of physiological motion effects in fMRI: RETROICOR. Magn Reson Med 2000; 44(1): 162-7.

[24] Zang Y, Jiang T, Lu Y, He Y, Tian L. Regional homogeneity approach to fMRI data analysis. Neuroimage 2004; 22(1): 394-400.

[25] Wu T, Long X, Zang Y, et al. Regional homogeneity changes in patients with Parkinson's disease. Hum Brain Mapp 2009; 30(5): $1502-10$

[26] Wu T, Wang L, Chen Y, Zhao C, Li K, Chan P. Changes of functional connectivity of the motor network in the resting state in Parkinson's disease. Neurosci Lett 2009; 460(1): 6-10.

[27] Raz N. Aging of the brain and its impact on cognitive performance: inte- gration of structural and functional findings. In: Salthouse FCTA, ed. The Handbook of Aging and Cognition, 2nd Edition Erlbaum, Hillsdale, NJ 2000; 1-90.

[28] Hyde JS, Biswal BB, Jesmanowicz A. High-resolution fMRI using multislice partial k-space GR-EPI with cubic voxels. Magn Reson Med 2001; 46(1): 114-25.

[29] Raichle ME, Mintun MA. Brain work and brain imaging. Annu Rev Neurosci 2006; 29: 449-76.

(C) Yan et al.; Licensee Bentham Open.

This is an open access article licensed under the terms of the Creative Commons Attribution Non-Commercial License (http://creativecommons.org/licenses/by-nc/3.0/) which permits unrestricted, non-commercial use, distribution and reproduction in any medium, provided the work is properly cited. 